GOSH lies in caring for patients up to 18 years, adolescent expertise is limited, as it is nationally. Conversely, UCLH has a unique expertise in complex and specialist adolescent care, which could assist the transition planning for those children with the most complex needs. Based on our data, children with motility and functional-GI disorders commonly require a transition plan spanning multiple adult services and require the expertise of the adolescent MDT including psychology, social work, youth work, psychiatry, physiotherapy and occupational therapy. A commissioned joint transition service between GOSH and UCLH would facilitate best practice and provide an exemplar of clinical care for young people with complex health problems.

\section{THE PREVALENCE OF COW'S MILK PROTEIN ALLERGY IN INFANTS WITH GASTROSCHISIS AND INTESTINAL FAILURE TYPE 2 AND 3}

Kornilia Nikaki, Tracey Johnson, Haidee Norton, Gabis Chana, Amrita Garcha, Michelle Butcher, Wolfram Haller, Lisa Whyte, Theodoric Wong, Sue Protheroe. Birmingham Children's Hospital

\subsection{6/flgastro-2021-bspghan.5}

Introduction The prevalence of cow's milk protein allergy (CMPA) in infants with gastroschisis has been reported as high as $45 \%$, which is significantly higher than in the general infant population $(0.5-1 \%$ of breast-fed babies and $5-7 \%$ in formula-fed babies). We aimed to define the prevalence of CMPA in infants with gastroschisis and type 2 or 3 intestinal failure (IF) and compare this to other groups of infants with type 2 or 3 IF.

Methods We obtained the pharmacy records of PN prescription lasting more than 28 days (i.e. IF type 2 and 3) for infants born between July 2015 and June 2020 in our tertiary intestinal rehabilitation centre. We only included infants presenting in the first year of life with type 2 or 3 IF related to a gastrointestinal disorder, other than enteropathies. We recorded the underlying cause of IF and the number of patients clinically diagnosed with CMPA. The diagnosis of CMPA was made by the clinical team on the basis of gastrointestinal symptoms, presence of macroscopic blood in the stools and lack of any other alternative diagnosis with symptom resolution after initiation of a hydrolysed or amino acid-based formula. We then obtained the feeding records of the patients and noted the number of infants on a hydrolysed and amino acid-based formula during their hospitalisation and at the latest dietetic follow up.

Results Out of 112 infants, 23 were diagnosed with gastroschisis and 29 with necrotising enterocolitis (NEC); 25/29 surgically managed. CMPA was diagnosed in $3 / 23$ (13\%) infants with gastroschisis, $5 / 25(20 \%)$ of infants with surgically managed NEC and 5/60 (8\%) of infants with other causes of IF type 2 and 3 . Out of 23 patients with gastroschisis, only one was discharge home on PN with no concerns of CMPA. Out of the total 112 infants, 98 presented with IF type 2 and only $7(7 \%)$ were also diagnosed with CMPA, while 14 were discharged on home PN with 6 (43\%) also diagnosed with CMPA - table 1. An amino acid-based formula was trialled in $30 / 112(27 \%)$ infants at some point during their hospitalisation while a hydrolysed formula was used in 54/112 (48\%) infants with type 2 and $3 \mathrm{IF}$, in order to treat fat and sugar malabsorption, with $57 \%$ of children continuing to take a

\begin{tabular}{lllll} 
Abstract 05 Table 1 & & & & \\
\hline & $\begin{array}{l}\text { No of } \\
\text { patients }\end{array}$ & CMPA & IF type 3 & $\begin{array}{l}\text { CMPA \& } \\
\text { IF type 3 }\end{array}$ \\
\hline Gastroschisis & 23 & 3 & 1 & 0 \\
Surgically managed NEC & 25 & 5 & 6 & 2 \\
Medically managed NEC & 4 & 0 & 0 & 0 \\
Intestinal atresia & 18 & 3 & 3 & 3 \\
Malrotation & 10 & 1 & 2 & 1 \\
Hirschprung's disease & 3 & 1 & 1 & 0 \\
Other & 29 & 0 & 1 & \\
Total & 112 & 13 & 14 & 6 \\
\hline
\end{tabular}

hydrolysed formula at the last recorded dietetic follow up. Three out of the 13 children with CMPA (2 with gastroschisis) have tolerated a dairy containing diet later on.

Conclusions The prevalence of CMPA in infants with gastroschisis and type 2 or 3 IF is much lower than that previously reported. CMPA is most prevalent in infants with surgically managed NEC and IF 2 or 3 while the prevalence of CMPA in IF type 3 in general is significantly higher. The use of hydrolysed formula for the management of malabsorption may be masking the diagnosis of CMPA in children with IF type 2.

\section{DELIVERY OF A NATIONAL PAEDIATRIC GASTROENTEROLOGY, HEPATOLOGY AND NUTRITION VIRTUAL TEACHING PROGRAMME DURING THE COVID- 19 PANDEMIC}

${ }^{1}$ Kwang Yang Lee, ${ }^{2}$ Neil McConnell, ${ }^{3}$ Rulla Al-Araji, ${ }^{4}$ Kavitha Jayaprakash, ${ }^{5}$ Robert Hegarty, ${ }^{3}$ Chayarani Kelgeri, ${ }^{3}$ Theodoric Wong, ${ }^{3}$ Sue Protheroe. ${ }^{1}$ Bristol Royal Hospital for Children; ${ }^{2}$ Royal Hospital for Sick Children, Glasgow; ${ }^{3}$ Birmingham Children's Hospital; ${ }^{4}$ Leeds Children's Hospital; ${ }^{5}$ King's College Hospital

\subsection{6/flgastro-2021-bspghan.6}

Introduction At the onset of the Covid-19 pandemic, hospital educational activities were halted in order to focus on healthcare delivery and maintain social distancing. As a response to this disruption, BSPGHAN trainees set up the BSPGHAN Education Series, a twice- weekly virtual learning programme. The core objective of this programme was to deliver high quality paediatric gastroenterology, hepatology and nutrition (PGHAN) teaching during the pandemic.

In this study, we analysed the attendances and feedback received from the education series, in order to guide future directions.

Methods We reviewed the Zoom meeting attendance logs and Survey Monkey feedback forms for the BSPGHAN Education Series from April 2020 to December 2020.

Results In nine months, a total of 55 talks were delivered by 43 speakers. $23(41.8 \%)$ sessions were gastroenterologythemed, 25 (45.4\%) were hepatology-themed and $7(12.7 \%)$ were nutrition-themed.

Thirteen paediatrics gastroenterology units (12 in the UK and 1 in the United States) and all 3 UK tertiary paediatric liver centres contributed to the talks. The highest contributing centres were Birmingham Children's Hospital (20 sessions), followed by King's College Hospital (9 sessions) and Leeds Children's Hospital (7 sessions). 